\title{
A case report of Madelung's disease
}

\author{
Nam Jang, \\ Hyun Woo Shin, \\ Junekyu Kim, \\ Kun Chul Yoon \\ Department of Plastic and \\ Reconstructive Surgery, Kangbuk \\ Samsung Hospital, Sungkyunkwan \\ University School of Medicine, Seoul, \\ Korea
}

Madelung's disease (MD) otherwise known as Launois-Bensaude syndrome, multiple symmetrical lipomatosis, or benign symmetric lipomatosis, is a rare disease characterized by abnormal diffuse lipomatosis in proximal upper limbs and neck. Here, we report a rare case of MD. A 66-year-old man presented with massive growth of soft tissues in the cervico-occipital region of more than 2 years duration. Physical examination showed diffuse enlargement of the anterior neck (Madelung's collar) and three huge humps at the posterior neck. Under a diagnosis of MD, lipectomy via a single anterior transverse incision and liposuction were performed. This rare case report may be helpful for assessing patients with abnormal diffuse lipomatosis in the neck and proximal upper limbs.

Keywords: Lipoma / Lipomatosis / Lipomatosis, multiple symmetrical

\section{INTRODUCTION}

Madelung's disease (MD) is a rare disease characterized by abnormal diffuse lipomatosis in proximal upper limbs and neck. In most patients, neck involvement results in a specific sign called Madelung's collar or horse collar [1,2]. The disorder was first described by Brodie in 1846, and subsequently, Madelung in 1888 and Launois and Bensaude in 1898 characterized the disease. MD has multiple synonyms, which include LaunoisBensaude syndrome, multiple symmetrical lipomatosis, and benign symmetric lipomatosis. Reportedly, its prevalence is 1:25,000 and it mainly affects males from the Mediterranean region (male: female ratio of 15-30:1), though a few Asian cases have been reported $[3,4]$. The etiology of MD remains unknown, but abnormal lipogenesis induced by catecholamines has been suggested [5]. In $60 \%$ to $90 \%$ of patients, MD is associated with chronic alcoholism $[1,6]$, though the causal relationship between alcohol intake and MD is unclear. Diagnosis is established through clinical history taking and physical ex-

\footnotetext{
Correspondence: Hyun Woo Shin

Department of Plastic and Reconstructive Surgery, Kangbuk Samsung Hospital, Sungkyunkwan University School of Medicine, 29 Saemunan-ro, Jongno-gu, Seoul 03181, Korea

E-mail: shw.shin@samsung.com

Received July 7, 2020 / Revised August 24, 2020 / Accepted October 8, 2020
}

amination, though imaging studies may contribute to differential diagnoses. In this case report, we present a case of MD and share our experience of treatment.

\section{CASE REPORT}

A 66-year-old man presented with massive growth of soft tissues in the cervico-occipital region, which had occurred over a period of more than 2 years (Fig. 1). He was a severe alcoholic but had no relevant medical history. Physical examination revealed diffuse enlargement of the anterior neck (Madelung's collar) and three huge humps at the posterior neck. Endocrine test results were normal, and laboratory testing, which included lipid profile, detected no abnormality. Preoperative magnetic resonance imaging (MRI) was performed to differentiate it from other diseases, such as morbid obesity, neurofibromatosis, Cushing syndrome, and lymphoma (Fig. 2), and showed symmetric massive fatty proliferation of temporal and occipital regions and of neck levels I-III and V and posterior neck, which were consistent with a diagnosis of MD.

Operative treatment was planned to address the bulging neck contour. No medical treatment protocol was applied because the patient did not exhibit any endocrine or laboratory abnormality. Surgical treatment included anterior and posterior neck 
liposuction and anterior neck lipectomy. Initially, liposuction was performed via slit incisions on the anterior, both lateral sides, and on the posterior neck, and then subcutaneous dissection and power-assisted liposuction were used to remove deep fatty tissues. Next, a wide, single, transverse incision (about 15 $\mathrm{cm}$ long) was made on the anterior neck, and deep and superficial fatty masses were partially excised (Fig. 3). Surgery was completed by trimming remnant skin and wound closure. Re-
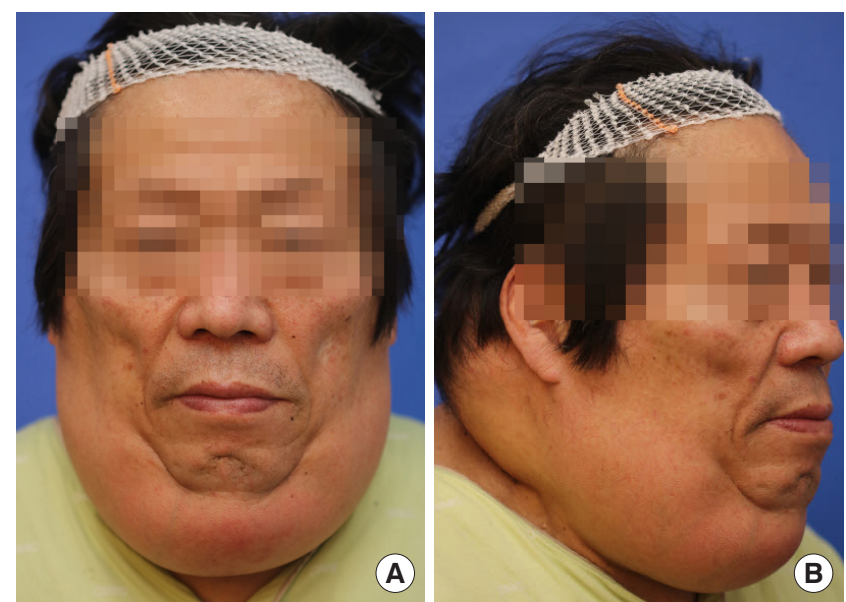

Fig. 1. (A, B) Preoperative images of a 66-year-old man with anterior and posterior cervical lipomatosis.

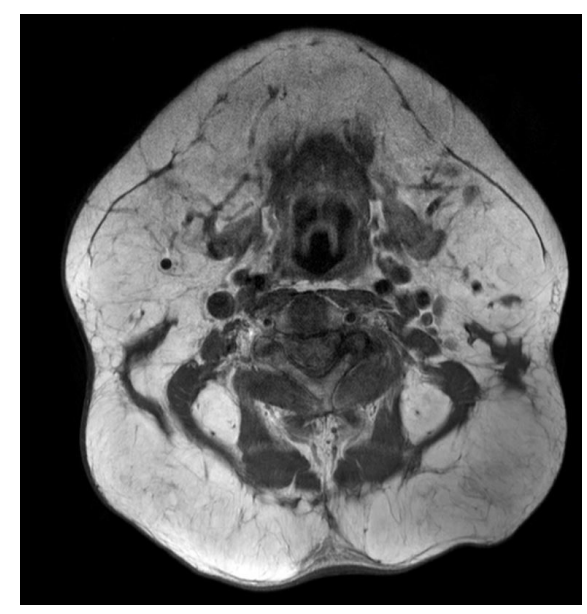

Fig. 2. Preoperative magnetic resonance image showing symmetric massive fatty proliferation at temporal and occipital regions, at neck levels I-III, V, and the posterior neck, which were consistent with Madelung's disease. covery was optimal and the patient was discharged without complication.

At his postoperative 2-month follow-up, although complete excision of adipose masses was not achieved, the patient showed improved appearance. No recurrence was evident during 1 year of follow-up and we planned a secondary operation, but the patient refused.

\section{DISCUSSION}

$\mathrm{MD}$ is a rare disorder characterized by the presence of multiple, symmetric, nonencapsulated fat masses in the face, neck, and other areas. Two different classifications of MD are used in the literature. Enzi's classification [7], which is based on anatomic fat distribution, classifies MD into two types. In type 1, fat distribution is symmetric and concentrated in the neck, shoulders, supraclavicular triangle and proximal upper limbs, whereas in type 2, the neck and upper trunk are not affected and deposits occur in abdomen and thighs, and thus, this type may be easily confused with normal obesity. In a more recent classification, Donhauser et al. [8] subdivided Enzi type 1 and defined three types of MD: type 1-neck distribution, type 2-a pseudoathletic appearance, and type 3-a gynecoid appearance (Table 1).

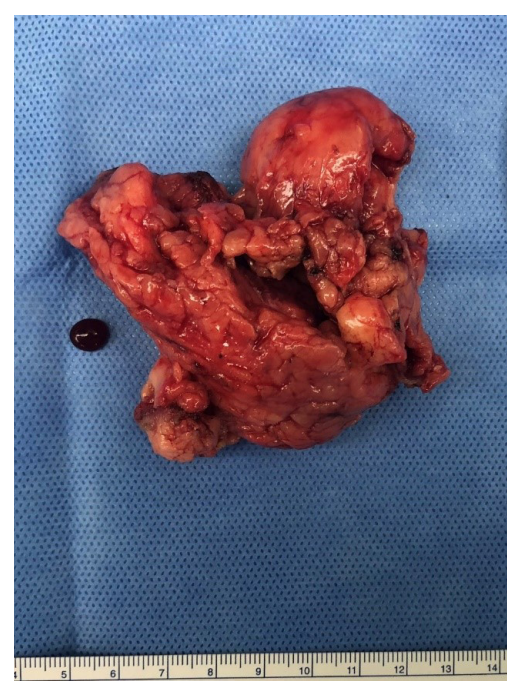

Fig. 3. Anterior neck lipectomy was performed and large fatty masses were partially excised (size: about $8 \times 7 \mathrm{~cm}$ ).

Table 1. Enzi and Donhauser classification

\begin{tabular}{lll}
\hline Enzi & \multicolumn{1}{c}{ Donhauser } & \multicolumn{1}{c}{ Affected body areas } \\
\hline Type 1 & Type I (horse collar) & Neck, upper back, shoulder girdle, and upper arms \\
& Type II (pseudo athletic type) & Shoulder girdle, deltoid region, upper arms, and thorax \\
& Type III (gynecoid type) & Lower body, especially the thighs and medial side of the knees \\
Type 2 & Type IV (abdominal type) & Abdomen \\
\hline
\end{tabular}


However, it should be noted that more than one type of distribution may be present in some patients. Our reported case was classified as Donhauser type $1 \mathrm{MD}$.

The pathophysiology of MD still remains unclear. Different theories have been proposed: a defect in the lipolytic pathway in response to catecholamines [5], mutations or deletions of mitochondrial DNA [9]. MD is associated with chronic alcoholism in $60 \%$ to $90 \%$ of the patients, though the causal relationship between alcohol and MD is still unclear [1].

A diagnosis of MD may be established based on clinical findings of multiple, symmetric fatty accumulations, typically distributed in the neck and proximal upper trunk, and relatively little fatty accumulation elsewhere. Imaging modalities such as computed tomography, ultrasonography, or MRI may aid differential diagnosis. In MD, fatty accumulations are non-capsulated and distributed along vascular and muscular planes, whereas in lipoma, they are encapsulated in subcutaneous space. Other disease entities such as angiolipoma, neurofibroma, liposarcoma and lipoblastomatosis, lipodistrophy, lymphoma, neurofibromatosis, and salivary gland disorders should also be considered, because, despite the benign nature of $\mathrm{MD}$, elimination of these entities enables adequate management.

We concur with most authors that the surgical approach is the best management option, though because the etiology of MD is unclear, this is viewed as palliative surgery performed for mainly cosmetic reasons. Surgical treatments include lipectomy and liposuction and the surgical technique chosen depends on fat deposit volume and location and surgeon's preference. Lipectomy enables thorough excision but requires correct identification of important structures and careful hemostasis. This technique is associated with low morbidity, and fatty masses can be exposed despite deep infiltration. Liposuction techniques are of limited efficacy for the treatment of large masses, but liposuction is much less invasive, has a lower complication rate, and leaves no scars $[4,10]$.

In the described case, we used lipectomy via a single anterior transverse incision, which was introduced by Wong et al. [11], and added liposuction at the anterior and posterior neck to eliminate adipose masses as much as possible and improve contour and symmetry. However, fat infiltrations in our patient were diffusely distributed, even near nerves and vessels, and boundaries with normal structures were unclear. Due to the risk of damaging important structures, such as spinal accessory nerves and external carotid artery and vein, it was difficult to totally excise fat infiltrations. In addition, fat depositions were surrounded by fibrous septa, which resulted in more bleeding and made liposuction more difficult than normally experienced in lipoma or obese patients.
For these reasons, the authors' treatment method that mixed lipectomy via a single anterior transverse incision and liposuction resulted in undercorrection. Accordingly, we suggest further studies on MD treatment methods be undertaken to avoid undercorrection and injury to important structures.

\section{NOTES}

\section{Conflict of interest}

No potential conflict of interest relevant to this article was reported.

\section{Ethical approval}

The study was approved by the Institutional Review Board of Kangbuk Samsung Medical Center (IRB No. KBSMC 2020-06047) and performed in accordance with the principles of the Declaration of Helsinki. Written informed consent was obtained.

\section{Patient consent}

The patient provided written informed consent for the publication and the use of his images.

\section{ORCID}

Nam Jang

Hyun Woo Shin

Junekyu Kim

Kun Chul Yoon

\section{REFERENCES}

1. Gonzalez-Garcia R, Rodriguez-Campo FJ, Sastre-Perez J, Munoz-Guerra MF. Benign symmetric lipomatosis (Madelung's disease): case reports and current management. Aesthetic Plast Surg 2004;28:108-12.

2. Verna G, Kefalas N, Boriani F, Carlucci S, Choc I, Bocchiotti MA. Launois-Bensaude syndrome: an unusual localization of obesity disease. Obes Surg 2008;18:1313-7.

3. Jimenez Aragon F, Morales Puebla JM, Corzon Pereira T. Madelung's disease. Acta Otorrinolaringol Esp 2013;64:166-7.

4. Pinto CI, Carvalho PJ, Correia MM. Madelung's disease: revision of 59 surgical cases. Aesthetic Plast Surg 2017;41:359-68.

5. Enzi G, Inelmen EM, Baritussio A, Dorigo P, Prosdocimi M, Mazzoleni F. Multiple symmetric lipomatosis: a defect in adrenergic-stimulated lipolysis. J Clin Invest 1977;60:1221-9.

6. Brea-Garcia B, Cameselle-Teijeiro, J, Couto-Gonzalez, I, Taboada-Suarez, A, Gonzalez-Alvazera, E. Madelung's disease: comorbidities, fatty mass distribution, and response to treat- 
ment of 22 patients. Aesthetic Plast Surg 2013;37:409-16.

7. Enzi G, Busetto L, Ceschin E, Coin A, Digito M, Pigozzo S. Multiple symmetric lipomatosis: clinical aspects and outcome in a long-term longitudinal study. Int J Obes Relat Metab Disord 2002;26:253-61.

8. Donhauser G, Vieluf D, Ruzicka T, Braun-Falco O. Benign symmetric Launois-Bensaude type III lipomatosis and BureauBarrière syndrome. Hautarzt 1991;42:311-4.

9. Plummer C, Spring PJ, Marotta R, Chin J, Taylor G, Sharpe D, et al. Multiple symmetrical lipomatosis: a mitochondrial disorder of brown fat. Mitochondrion 2013;13:269-76.

10. Kim JH, Lee YJ, Oh DY. Minimal scarring from giant intramuscular lipoma excision with the harmonic scalpel. Arch Aesthetic Plast Surg 2019;25:159-62.

11. Wong DS, Lam LK, Chung JH, Ng RW, Li GK, Chan VS. Aesthetic considerations in the cervicofacial management of Madelung syndrome. Scand J Plast Reconstr Surg Hand Surg 2003;37:34-40. 